\title{
Amoebic Liver Abscess and Indigenous Alcoholic Beverages in the Tropics
}

\author{
T. Kumanan $\left(\mathbb{1},{ }^{1}\right.$ V. Sujanitha $(1),{ }^{1}$ S. Balakumar, ${ }^{2}$ and N. Sreeharan ${ }^{1}$ \\ ${ }^{1}$ Department of Medicine, Faculty of Medicine, University of Jaffna, Jaffna, Sri Lanka \\ ${ }^{2}$ Department of Biochemistry, Faculty of Medicine, University of Jaffna, Jaffna, Sri Lanka \\ Correspondence should be addressed to V. Sujanitha; sujanitha@yahoo.com
}

Received 14 March 2018; Accepted 25 June 2018; Published 10 July 2018

Academic Editor: Pedro P. Chieffi

Copyright (c) 2018 T. Kumanan et al. This is an open access article distributed under the Creative Commons Attribution License, which permits unrestricted use, distribution, and reproduction in any medium, provided the original work is properly cited.

\begin{abstract}
Amoebic liver abscess (ALA) seen commonly in the tropics is predominantly confined to adult males, especially those who consume locally brewed alcohol, although intestinal amoebiasis occurs in all age groups and in both genders. Whether the role of alcohol in the development of ALA is incidental and casual or whether alcohol is causally implicated has been debated. It has been argued that socioeconomic factors and poor sanitary conditions are the primary culprits that casually link alcohol to ALA. However, there has emerged an abundance of data that implicates alcohol in a more causal role in facilitating the extraintestinal invasion of the infective protozoan and the subsequent development of ALA. These factors include the role of alcohol in host immunity, parasitic proliferation, and invasion and in creating a conducive hepatic microenvironment. The contributory role of alcoholinduced increase in hepatic iron stores and lipid content is discussed. Late-stage liver disease with fibrosis seems to be protective for the development of ALA. Further research is necessary to elucidate the many possible mechanisms that predispose to hepatic amoebiasis, so that appropriate individual and population-based preventive measures can be implemented.
\end{abstract}

\section{Introduction}

Liver abscess may result from a variety of pathogenic organisms including bacteria, fungus, protozoa, and parasites. It is a common clinical entity encountered by physicians throughout the world. One of the early reports states that two-thirds of its aetiology in the tropics could be attributed to amoebic liver abscess (ALA); in contrast, three-fourths in the developed countries are attributed to bacterial pathogens [1].

ALA is very common in many Asian countries including Sri Lanka. Incidence of ALA in other continents is mainly due to Entamoeba histolytica infection acquired during travelling. The prevalence of ALA in different places across the world is shown in Table 1.

ALA caused by Entamoeba histolytica is presently the third most common cause of death from parasitic diseases [2]. Susceptibility to this infection and the clinical outcomes are variable in the vulnerable population. Most of the studies from tropics illustrate the fact that ALA is extremely common among males who consume alcohol beverages, in particular the indigenous variety. This interesting and important association raises a query: Is this relationship casual or causal?

\section{Amoebic Liver Abscess and Alcohol}

A close relationship between the consumption of indigenously brewed alcohol beverages and the occurrence of ALA in the tropics has been established over a period of time by several large-population studies [3-5]

Reasons for this association between local alcohol beverages and ALA could be multifactorial. Factors influencing the association could be related to the pathogen, contents of beverages, status of the liver, and the immunity of the host. They are more vulnerable perhaps due to the large infective dose of Entamoeba histolytica and other bacterial pathogens ingested with the unhygienically brewed beverage. Associated nutritional status of the population and poor sanitation could also play a pivotal role. Hai et al. stated that alcohol-induced hepatic dysfunction and possible suppression of amoebistatic immune mechanisms by substances in the beverages could also be attributed in the mechanism [6]. 
TABLE 1: Prevalence of ALA in different places across the world.

\begin{tabular}{lcccc}
\hline Region/country & Origin & Period & Total number of ALA cases & References \\
\hline Bangladesh & Hospitals in northern district & $2008-2010$ & 90 & Alam et al. 2014 [10] \\
Sri Lanka & Teaching Hospital-Jaffna & $2012-2015$ & 367 & Kannathasan et al. 2018 [3] \\
Bordeaux, France & Bordeaux University Hospital Centre & $1995-1999$ & $20(18$ HIV+) & Djossou et al. 2003 [42] \\
Thailand & Nationwide hospital admission data & $2008-2013$ & 448 & Poovorawan et al. 2016 [43] \\
Toronto, Canada & Seven hospitals in Toronto & $1980-2005$ & 29 & Wuerz et al. 2012 [44] \\
Sonora, Mexico & 4 hospitals in Hermosillo & $2000-2005$ & 319 & Valenzuela et al. 2007 [45] \\
San Francisco, USA & Medical histories & $1979-1994$ & 56 & Seeto and Rockey 1999 [46] \\
\hline
\end{tabular}

Mukhopadhyay et al. [5] have suggested that "alcohol can predispose to ALA through a multitude of mechanisms, including hepatic damage by alcohol, lowered body resistance and suppression of liver function due to poor nutritional status of habitual consumers of alcohol, increased presence of amoebae in the liquor prepared locally with poor regard to aseptic procedures, and depression of immune mechanisms in chronic alcoholics." A study conducted in India in 2011 [4] showed that $67.5 \%$ of patients with amoebic liver abscess are from the low socioeconomic class and $72 \%$ were alcoholics. It was also noted that alcoholics had larger abscesses, a greater frequency of complications, and delayed resolution of the abscesses.

2.1. Age and Gender Differences in Amoebic Liver Abscess. Many studies have confirmed the fact that ALA is extremely common in adult males. Naturally the question whether this could be explained by the gross cultural disparity observed in drinking habits between adult males, females, and children or whether other possible aetiological mechanisms exist to explain the gender difference arises.

Amoebic liver abscess is extremely rare in children and infection is mainly restricted to the gut [7]. Malnutrition predisposes children to infection and the nutritional hormone leptin is important in the immunity to the enteric pathogen Entamoeba histolytica [8]. Malnourished children are known to have low levels of the hormone leptin that signals satiety and also influences the immune system. Genetic variants in leptin and the leptin receptor were evaluated for association with infection, and increased susceptibility to intestinal Entamoeba histolytica infection was found to be associated with a single amino acid polymorphism in the leptin receptor in the children studied in a Bangladeshi cohort of amoebiasis [9].

Although intestinal amoebiasis and asymptomatic amoebic infections are common among children and females, why ALA is extremely uncommon in these population is unanswered and a matter of debate.

The incidence of amoebic liver abscess in males increases after puberty and the peak incidence is approximately at the age of 40 years [7]. A study was done to examine the gender differences in immunocompetent mice to amoebic infection of the liver and found that $75 \%$ of male mice developed abscess, while none of the female mice did so. It also revealed that female mice cleared the infection faster and there were clear differences in the production of cytokines in both categories, which could explain the differences in the infection rate in both genders [7]. No human studies have been done to elicit this gender difference to date.

Several studies have highlighted the strong association of alcohol intake, mainly of the indigenous variety, in the preponderance of males developing ALA. A study in Bangladesh found that the male-to-female ratio was 21:1 and a habit of indigenous alcohol consumption was observed in four-fifths of the patients with ALA [10]. A Sri Lankan study recently found that almost all the 346 confirmed ALA patients were males and consumed alcohol beverages, in particular the indigenous variety [11]. Similar observation was made in an Indian study [6] conducted in the early 80s among 220 patients with ALA. The majority of the subjects were young or middle-aged males and $85 \%$ gave a history of drinking toddy, a local palm wine of the region.

However, Reddy and Thangavelu [12] proposed alternative mechanisms to explain the gender difference, suggesting that "the female menstrual cycle prevents hepatic congestion and thus makes the organ less susceptible to abscess formation. Additionally, a relative iron deficiency due to menstruation and protective hormonal factors in women of child bearing age limits Entamoeba histolytica invasion in females." A laboratory-based study [13] found that testosterone increased the susceptibility of mice to Entamoeba histolytica liver abscess by reducing the interferon-gamma secretion by natural killer cells. Therefore, these alternative, non-alcohol-based mechanisms could also explain the relative preponderance of ALA in males.

2.2. Amoebic Liver Abscess and Alcohol: Is It a Casual Association? We will initially explore the factors that point towards a casual relationship with alcohol and other factors, primarily related to socioeconomic status and personal hygiene possibly being the primary culprits. As most of the studies clearly point out, ALA almost always occurs in males with a history of drinking indigenously brewed alcohol beverages in a poor socioeconomic background. As the primary contributory factor is the male gender, we need to explore whether this gender preponderance is purely due to alcohol-related factors or whether it is due to other factors including the level of immunity of the host.

How amoeba gets into the beverage is not well understood. Most likely this is due to the unhygienic practices commonly seen at the drinking taverns or cottages in many tropical countries. The important finding observed in most of 
the studies is that majority of ALA patients are from a poor socioeconomic background and are manual laborers [6, 10, 11]. Poor hygiene has been associated with increased risk of amoebic liver abscesses and is directly proportionate to disease progression and extent of liver injury [14]. Parasite cysts are transmitted through contaminated food and water, making the incidence of disease high in areas of poor sanitation. Good hygienic practices cannot be expected at these places as the majority of subjects are male farmers from lowerand middle-class backgrounds in one study [15] and $68 \%$ belonged to low socioeconomic group in another study [11]. The prevalence of asymptomatic intestinal amoebiasis is also common in these regions, although the prevalence rate varies with the methods used for screening. It is high when microscopic examination method is employed and low with the use of new molecular techniques [16]. It can be assumed that the regular visitors to these drinking places could be the source of pathogen by unhygienic practices including open air defecation, poor hand washing practices, unwashed utensils at these taverns, and the consumption of unboiled drinking water. Indeed, some studies have documented the prevalence of vectors of amoebiasis, including flies and cockroaches at these places [17]. The study done in northern Sri Lanka [11] showed heavy alcohol consumption, mainly toddy, in all 346 confirmed patients with amoebic liver abscesses but none of the cultured toddy samples grow Entamoeba histolytica.

All the above considerations may suggest that the relationship between alcoholic beverages and ALA is incidental and casual.

2.3. Alcohol as a Causal Factor of Amoebic Liver Abscess. We now turn to the accumulation of evidences which suggests that the relationship between alcohol consumption and ALA is not incidental and casual but causal in nature. The beverage and its constituent ethanol could influence several factors to make individuals infected with amoeba to develop extraintestinal invasion. These factors could be pathogen- or hostrelated.

2.4. Alcohol and Immunity. Immunological mechanisms of the host are important in combating a protozoal pathogen. The extensive nature of amoebic liver abscess in patients with HIV infection proves that cell-mediated immunity is very important in controlling the disease [18]. Furthermore, animal studies have shown that immune suppression after thymectomy or splenectomy resulted in an increased incidence of amoebic liver abscess [19].

Alcohol has a significant influence on both cell-mediated and humoral immunity. Ethanol abuse is an important cause for reactivation of tuberculosis in the tropics [20]. There is ample evidence to suggest that both acute alcohol consumption and chronic alcohol consumption suppress almost all the components of the immune system, including initial response to a pathogen and the tumor surveillance system [21-23]. Alcohol suppresses function of Kupffer cells in liver, which has important role in clearing the amoebae.

2.5. Alcohol and Entamoeba Proliferation. Entamoeba can be cultured in both xenic and axenic culture media. Boeck and
Drbohlav first introduced xenic culture of Entamoeba in 1925 in a diphasic egg slant medium, and a modification of this medium (Locke-egg) is still used today [24]. A culture of parasites grown in association with an unknown microbiota is called xenic culture. Whether palmyrah wine/toddy provided a similar medium for the proliferation of Entamoeba histolytica is yet to be established. It is reasonable to infer such a possibility as the constituents of palm wine provide ample nutrients in the form of disaccharides and enormous amount of yeast cells to feed on. The acetic acid could provide an optimum mild acidic $\mathrm{pH}$ for its optimum growth. Mortan et al. have found that the components of jiggery made up of palmyrah palm of northern Sri Lanka include " $1.04 \%$ protein, $0.19 \%$ fat, $76.86 \%$ sucrose, $1.66 \%$ glucose, $3.15 \%$ total minerals, $0.861 \%$ calcium, and $0.052 \%$ phosphorus and also $11.01 \mathrm{mg}$ iron per $100 \mathrm{~g}$ and $0.767 \mathrm{mg}$ of copper per $100 \mathrm{~g}$ " $[25,26]$. The alcohol content of toddy is $4.5-7 \%$. As palmyrah toddy contains both iron and alcohol, both these constituents can have a synergistic action to cause ALA. Therefore, palmyrah toddy provides a more favourable microenvironment than distilled alcoholic beverage, which has no iron in its composition, for the proliferation of amoeba, thereby facilitating the pathogenesis of ALA.

Alcohol-induced hyperpermeability and dysbiosis of intestinal bacteria may both contribute to the pathological effects of alcohol, permitting the entry of Entamoeba histolytica into the blood [27].

A study found that exacerbated inflammatory milieu is established and contributes to liver tissue damage and probably supports the survival of parasites. IL- $1 \beta$ was detected mainly in neutrophils and macrophages from the periods of acute infection to subacute and chronic infection and decreased when granuloma was formed [28].

Parasite genotype is also a contributory factor for the causation of amoebic liver abscess. Studies have confirmed that intestine and liver abscess amoebae are genetically distinct. This suggests that either Entamoeba histolytica subpopulation in the same infection shows varying organ tropism or DNA reorganization event takes place prior to or during metastasis from intestine to liver [29].

Several host-related factors could contribute to its invasiveness in the liver including the architecture, fat content, and hormones. Several other factors contribute to the pathogenicity of Entamoeba histolytica and some may still await identification. Three important pathogenic factors that have been investigated and characterized are at molecular level: (1) the Gal/GalNAc adhesion, mediating adherence to host cells and contributing to amoebic resistance to complement, (2) the amoebapores, small peptides that produce pores in target membrane, and (3) cysteine proteinases that play a key role in Entamoeba histolytica tissue invasion, evasion of host defence, and parasite induction of inflammation [30]. Beyond doubt Entamoeba histolytica genome sequence and new molecular techniques will rapidly advance the understanding of the epidemiology and pathogenicity of amoebiasis. And also further work needs to be done to see whether creating a microbiological and biochemical environment similar to palm toddy enhances the growth of Entamoeba histolytica in culture media. 
2.6. Alcohol and Liver. Although alcohol is consumed by many, serious alcoholic liver disease occurs in susceptible people only. The deleterious effects of alcohol on liver are influenced by heredity, gender, diet, and other coexisting liver diseases. Liver injury by alcohol is mainly due to its metabolites due to direct toxicity or by inflammation induced by them. These types of continuous liver injury can lead to fibrosis and cirrhosis later [31].

Several factors related to the status of liver could also contribute a favourable environment to the amoeba when it reaches the liver. It is interesting that only small numbers of amoeba have been identified relative to the size of the abscess, which suggests that Entamoeba histolytica can kill hepatocytes effectively with extensive tissue destruction [32]. Studies have pointed out that chronic stage of ALA is characterized by defective cell-mediated immunity and the suppression of $\mathrm{T}$ cells and their defective proliferative responses [33]. Experimental studies have revealed the fact that presence of iron promotes the growth of amoeba in vitro. [26] Chronic ethanol consumption has been well documented to increase the hepatic iron stores [34]. It is therefore fair to postulate that high hepatic iron content is an important contributory factor to develop ALA, although the exact mechanisms are yet to be established [35]. Possible mechanisms include hepcidin, a key hormone in iron metabolism which inhibits intestinal iron absorption and the release of iron from macrophages. Alcohol has been shown to downregulate hepcidin expression in the liver, leading to elevated expression of iron transporter proteins in the duodenum [34]. Furthermore, another study [36] demonstrated that iron chelation interrupts the completion of the fermentation pathway of Entamoeba histolytica by removing the metal cofactor indispensable for the functional stability of alcohol dehydrogenase, thus affecting trophozoite survival. Hepatic tissue injury is directly correlated to the duration and amount of iron loading. Iron is an important factor for Entamoeba histolytica growth and virulence and also an important factor in the adherence and cytotoxicity of Entamoeba histolytica, acting by modulation of gene expression of virulence factors $[37,38]$. Thus, the above ironbased mechanisms together with increased iron content of palmyrah toddy [25] as opposed to nontraditional alcoholic spirits would favour a specific causal role for the indigenous beverage to enhance hepatic iron content and provide a conducive environment for Entamoeba histolytica growth.

Another study found that the incidence of liver abscess in the cirrhotic individuals was low, despite their increased susceptibility to infections due to diminished immunity [39]. The postulated mechanisms are many. Entamoeba histolytica lacks mitochondria and hence survives by scavenging nutrients from the host cells. The organism is rich in phospholipases and lysophospholipase essential for cytolysis of cells [40]. Hypocholesterolemia has been recorded in patients with ALA [41]. Fatty Liver is high in intracellular triglycerides and is an organ active in the production of cholesterol. Cholesterol increases the virulence of Entamoeba histolytica in animal models and in vitro culture. It is also likely that as the fatty liver disease progresses to cirrhosis, the environment to favour residence and multiplication of Entamoeba histolytica is lost due to both loss of nutrients from the hepatocytes and the "loss of space" as a result of fibrotic scarring, hence the rarity of cirrhosis and ALA.

\section{Conclusion}

This review has highlighted the strong association between the consumption of alcohol, particularly of the indigenous variety such as palmyrah toddy, and the development of hepatic amoebiasis. Although the link with alcohol could be casual with other socioeconomic and personal hygienic factors contributing to this association, there appears to be overwhelming evidence to suggest a more causal role for alcohol. It is likely that alcohol creates an immune and biochemical microenvironment that enhances extraintestinal invasion and settlement in the liver of Entamoeba histolytica and transforms asymptomatic and symptomatic intestinal amoebiasis into hepatic amoebiasis, a disease that is common in the tropics and a major cause of morbidity and mortality. Since humans are the only significant reservoir of Entamoeba histolytica infection, it is essential to identify the modifiable causes especially in developing countries so that the disease burden can be reduced or even eliminated. Further research is necessary to elucidate the many possible mechanisms that predispose to hepatic amoebiasis so that appropriate individual and population-based preventive measures can be implemented.

\section{Conflicts of Interest}

The authors declare that there were no conflicts of interest in preparation of this article.

\section{References}

[1] A. Ochsner, M. DeBakey, and S. Murray, "Pyogenic abscess of the liver. II. An analysis of forty-seven cases with review of the literature," The American Journal of Surgery, vol. 40, no. 1, pp. 292-319, 1938.

[2] A. J. Greenstein, D. Lowenthal, G. S. Hammer, F. Schaffner, and A. H. Aufses, "Continuing Changing Patterns of Disease in Pyogenic Liver Abscess: A Study of 38 Patients," American Journal of Gastroenterology, vol. 79, no. 3, pp. 217-226, 1984.

[3] S. Kannathasan, A. Murugananthan, T. Kumanan et al., "Epidemiology and factors associated with amoebic liver abscess in northern Sri Lanka," BMC Public Health, vol. 18, no. 1, 2018.

[4] Soumik Ghosh, Sourabh Sharma, A. K. Gadpayle et al., "Clinical, Laboratory, and Management Profile in Patients of Liver Abscess from Northern India," Journal of Tropical Medicine, vol. 2014, 8 pages, 2014.

[5] M. Mukhopadhyay, A. K. Saha, A. Sarkar, and S. Mukherjee, "Amoebic liver abscess: Presentation and complications," Indian Journal of Surgery, vol. 72, no. 1, pp. 41-45, 2010.

[6] A. A. Hai, A. Singh, V. K. Mittal, and G. C. Karan, "Amoebic liver abscess. Review of 220 cases," International Surgery Journal, vol. 76, no. 2, pp. 81-83, 1991.

[7] H. Lotter, T. Jacobs, I. Gaworski, and E. Tannich, "Sexual dimorphism in the control of amebic liver abscess in a mouse model of disease," Infection and Immunity, vol. 74, no. 1, pp. 118124, 2006. 
[8] G. Vedantam and V. K. Viswanathan, "Leptin signaling protects the gut from Entamoeba histolytica infection," Gut Microbes, vol. 3, no. 1, 2012.

[9] P. Duggal, X. Guo, R. Haque et al., "A mutation in the leptin receptor is associated with Entamoeba histolytica infection in children," The Journal of Clinical Investigation, vol. 121, no. 3, pp. 1191-1198, 2011.

[10] F. Alam, M. Salam, P. Hassan, I. Mahmood, M. Kabir, and R. Haque, "Amebic liver abscess in northern region of Bangladesh: sociodemographic determinants and clinical outcomes," $B M C$ Research Notes, vol. 7, no. 1, p. 625, 2014.

[11] S. Kannathasan, A. Murugananthan, T. Kumanan et al., "Amoebic liver abscess in northern Sri Lanka: first report of immunological and molecular confirmation of aetiology," Parasites \& Vectors, vol. 10, no. 1, pp. 1-7, 2017.

[12] D. G. Reddy and M. Thangavelu, "Some aspects of amoebiasis in Madras," Indian Medical Gazette, vol. 83, no. 12, pp. 557-563, 1948.

[13] H. Lotter, E. Helk, H. Bernin et al., "Testosterone Increases Susceptibility to Amebic Liver Abscess in Mice and Mediates Inhibition of IFN $\gamma$ Secretion in Natural Killer T Cells," PLoS ONE, vol. 8, no. 2, p. e55694, 2013.

[14] F. Alam, A. Salam, and I. Mahmood, "ALA is associated with malnutrition and low serum leptin level," Journal of Infectious Diseases \& Therapy, vol. 4, no. 5, 2016.

[15] Q. Islam, A. Ekram, M. Ahmed et al., "Pyogenic Liver Abscess and Indigenous Alcohol," TAJ: Journal of Teachers Association, vol. 18 , no. $1,2009$.

[16] K. Khairnar, S. C. Parija, and R. Palaniappan, "Diagnosis of intestinal amoebiasis by using nested polymerase chain reaction-restriction fragment length polymorphism assay," Journal of Gastroenterology, vol. 42, no. 8, pp. 631-640, 2007.

[17] T. Janani, P. Pushpana, R. Surenthirakumaran, T. Kumanan, and S. Kannanthasan, "Amoebic liver abscess: An emerging threat in northern Sri Lanka," in EMBO global lecture course and symposium on amoebiasis: Exploring the biology and pathogenesis of Entamoeba, vol. 80, Khajuraho, India, 2011.

[18] W. B. Park, P. G. Choe, J. Hyun Jo et al., "Amebic Liver Abscess in HIV-infected Patients, Republic of Korea," Emerging Infectious Diseases, vol. 13, no. 3, pp. 516-517, 2007.

[19] E. Ghadirian and E. Meerovitch, "Effect of immunosuppression on the size and metastasis of amoebic liver abscesses in hamsters," Parasite Immunology, vol. 3, no. 4, pp. 329-338, 1981.

[20] J. Rehm, A. V. Samokhvalov, M. G. Neuman et al., "The association between alcohol use, alcohol use disorders and tuberculosis (TB). A systematic review," BMC Public Health, vol. 9, article no. 450, 2009.

[21] R. T. Cook, "Alcohol abuse, alcoholism, and damage to the immune system-a review," Alcoholism: Clinical and Experimental Research, vol. 22, no. 9, pp. 1927-1942, 1998.

[22] L. E. Díaz, A. Montero, M. González-Gross, A. I. Vallejo, J. Romeo, and A. Marcos, "Influence of alcohol consumption on immunological status: A review," European Journal of Clinical Nutrition, vol. 56, pp. S50-S53, 2002.

[23] S. Nelson and J. K. Kolls, "Alcohol, host defence and society," Nature Reviews Immunology, vol. 2, no. 3, pp. 205-209, 2002.

[24] C. G. Clark and L. S. Diamond, "Methods for cultivation of luminal parasitic protists of clinical importance," Clinical Microbiology Reviews, vol. 15, no. 3, pp. 329-341, 2002.

[25] J. F. Morton, "Notes on distribution, propagation, and products of Borassus Palms (Arecaceae)," Economic Botany, vol. 42, no. 3, pp. $420-441,1988$.
[26] "Symeonidis A and Marangos M. Iron and Microbial Growth," in Insight and Control of Infectious Disease in Global Scenario, 2012, ISBN: 978-953-51-0319-6.

[27] C. B. Forsyth, R. M. Voigt, and A. Keshavarzian, "Intestinal CYP2E1: A mediator of alcohol-induced gut leakiness," Redox Biology, vol. 3, pp. 40-46, 2014.

[28] J. Pacheco-Yépez, J. M. Galván-Moroyoqui, I. Meza, V. Tsutsumi, and M. Shibayama, "Expression of cytokines and their regulation during amoebic liver abscess development," Parasite Immunology, vol. 33, no. 1, pp. 56-64, 2011.

[29] I. K. M. Ali, S. Solaymani-Mohammadi, J. Akhter et al., “Tissue invasion by Entamoeba histolytica: Evidence of genetic selection and/or DNA reorganization events in organ tropism," PLOS Neglected Tropical Diseases, vol. 2, no. 4, article no. e219, 2008.

[30] S. Solaymani-Mohammadi and W. A. Petri, "Intestinal invasion by Entamoeba histolytica," Subcellular Biochemistry, vol. 47, pp. 221-232, 2008.

[31] J. J. Maher, "Exploring alcohols Effects on liver function," Alcohol health and research world, vol. 21, no. 1, p. 12, 1997.

[32] A. Samie, A. ElBakri, and R. AbuOdeh, "Amoebiasis in the Tropics: Epidemiology and Pathogenesis," in Current Topics in Tropical Medicine, A. J. Rodriguez-Morales, Ed., chapter 14, pp. 978-953, 2012, ISBN 978-953-51-0274-8.

[33] D. Campbell, D. Gaucher, and K. Chadee, "Serum from Entamoeba histolytica-infected gerbils selectively suppresses T cell proliferation by inhibiting interleukin-2 production," The Journal of Infectious Diseases, vol. 179, no. 6, pp. 1495-1501, 1999.

[34] B. Cylwik, L. Chroslek, and M. Szmitkowski, "The effect of alcohol in the regulation of iron metabolism," Pol Merkur Lekarski, vol. 25, no. 147, pp. 273-275, 2008.

[35] R. P. S. Makkar, G. K. Sachdev, and V. Malhotra, "Alcohol consumption, hepatic iron load and the risk of amoebic liver abscess: A case-control study," Internal Medicine, vol. 42, no. 8, pp. 644-649, 2003.

[36] A. Espinosa, G. Perdrizet, G. Paz-y-Miño C, R. Lanfranchi, and M. Phay, "Effects of iron depletion on Entamoeba histolytica alcohol dehydrogenase 2 (EhADH2) and trophozoite growth: implications for antiamoebic therapy," Journal of Antimicrobial Chemotherapy, vol. 63, no. 4, pp. 675-678, 2009.

[37] J. Lee, S.-J. Park, and T.-S. Yong, "Effect of iron on adherence and cytotoxicity of Entamoeba histolytica to CHO cell monolayers," The Korean Journal of Parasitology, vol. 46, no. 1, pp. 37-40, 2008.

[38] L. Soto-Castro, L. Y. Plata-Guzmán, E. E. Figueroa-Angulo et al., "Iron Responsive-Like elements in the parasite entamoeba histolytica," Microbiology (United Kingdom), vol. 163, no. 9, pp. 1329-1342, 2017.

[39] C.-M. Kuo, C.-H. Kuo, and C.-S. Changchien, "Liver abscess in patients with cirrhosis of the liver: A 12-year experience," Journal of Gastroenterology, vol. 36, no. 8, pp. 552-556, 2001.

[40] S. A. Long-Krug, K. J. Fischer, R. M. Hysmith, and J. I. Ravdin, "Phospholipase a enzymes of entamoeba histolytica: Description and subcellular localization," The Journal of Infectious Diseases, vol. 152, no. 3, pp. 536-541, 1985.

[41] M. S. Flores, A. Obregón-Cárdenas, and E. Tamez, "Hypocholesterolaemia in patients with ALA," Gut Liver, vol. 8, no. 4, pp. 415-420, 2014.

[42] F. Djossou, D. Malvy, M. Tamboura et al., "Amoebic liver abscess. Study of 20 cases with literature review," La Revue de Médecine Interne, vol. 24, no. 2, pp. 97-106, 2003. 
[43] K. Poovorawan, W. Pan-Ngum, N. Soonthornworasiri et al., "Burden of liver abscess and survival risk score in Thailand: A population-based study," The American Journal of Tropical Medicine and Hygiene, vol. 95, no. 3, pp. 683-688, 2016.

[44] T. Wuerz, J. B. Kane, A. K. Boggild et al., "A Review of Amoebic Liver Abscess for Clinicians in a Nonendemic Setting," Canadian Journal of Gastroenterology \& Hepatology, vol. 26, pp. 1-5, 2012.

[45] O. Valenzuela, P. Moran, and A. Gomez, "Epidemiology of amoebic liver abscess in Mexico: the case of Sonora," Annals of Tropical Medicine Parasitology, vol. 101, no. 6, pp. 533-538, 2007.

[46] R. K. Seeto and D. C. Rockey, "Amoebic liver abscess: epidemiology, clinical features, and outcome," Western Journal of Medicine, vol. 170, no. 2, pp. 104-109, 1999. 


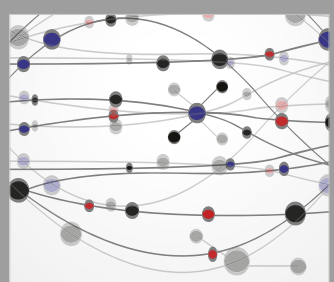

The Scientific World Journal
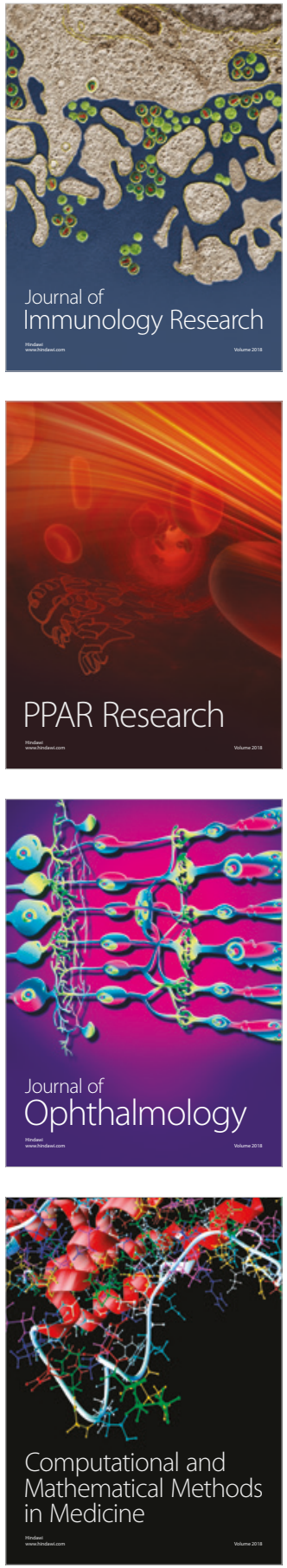

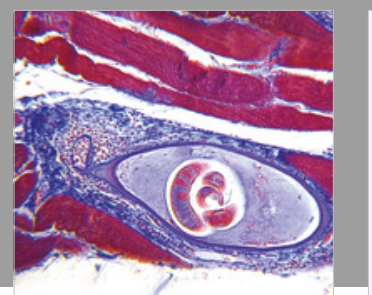

Gastroenterology Research and Practice

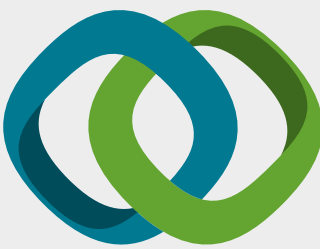

\section{Hindawi}

Submit your manuscripts at

www.hindawi.com
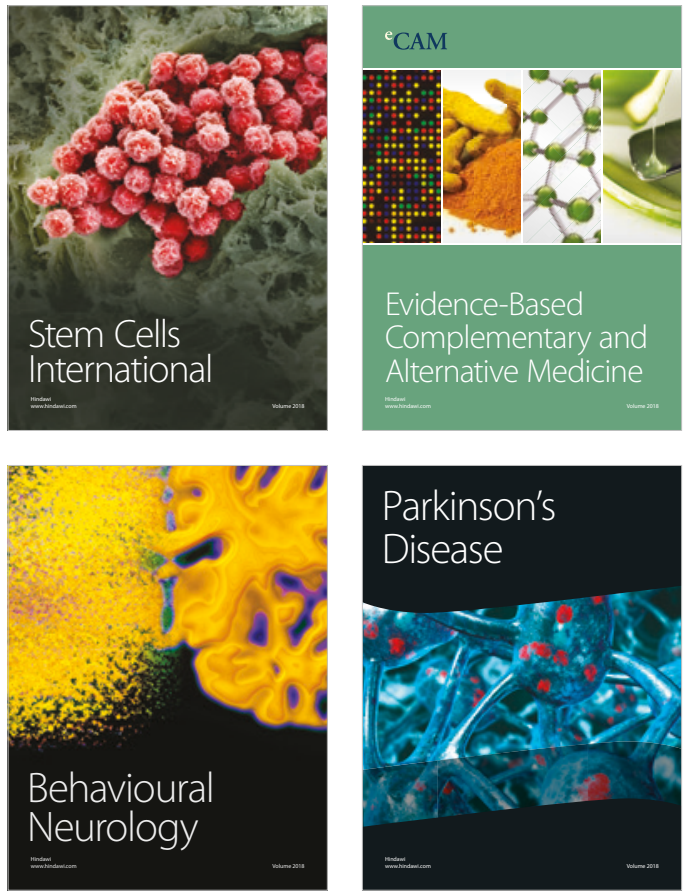

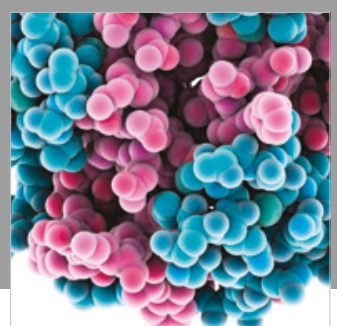

ournal of

Diabetes Research

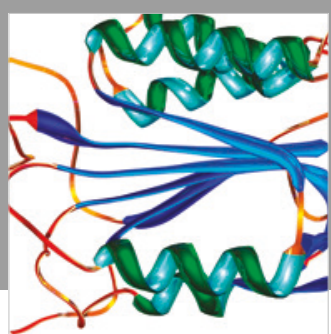

Disease Markers
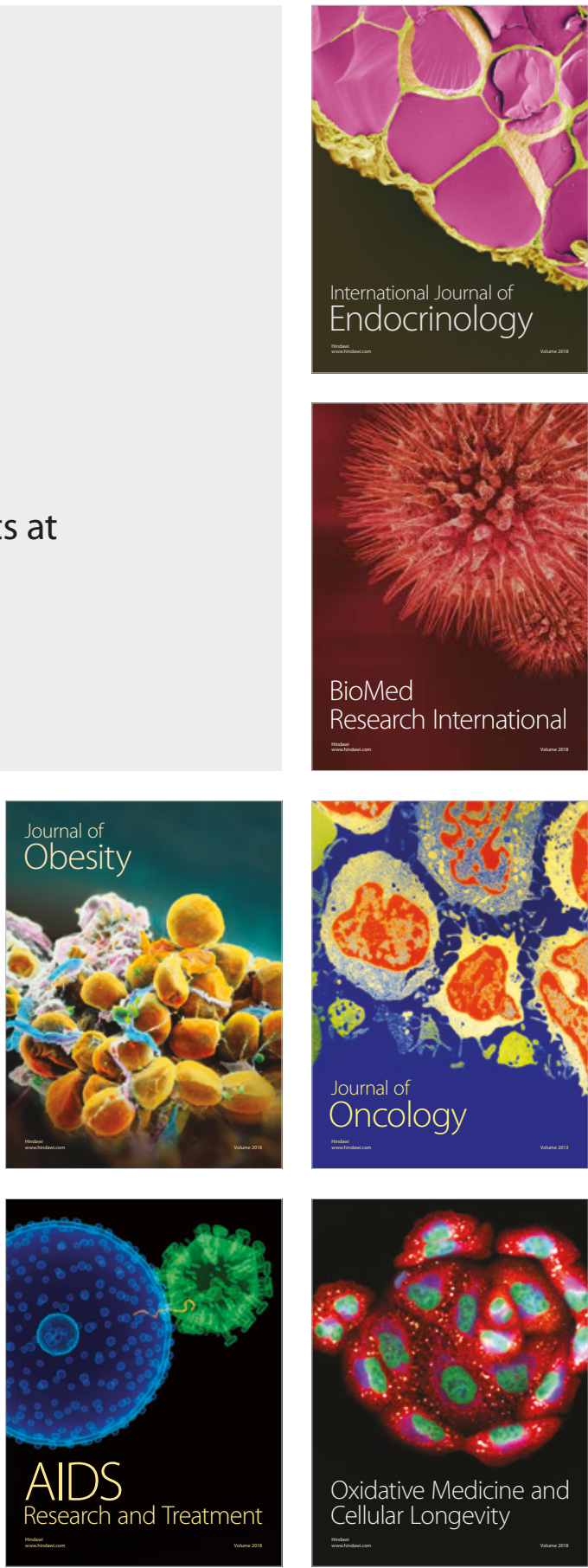